\title{
Pinning a Line by Balls or Ovaloids in $\mathbb{R}^{3}$
}

\author{
Xavier Goaoc • Stefan König • Sylvain Petitjean
}

Received: 27 January 2010 / Revised: 21 July 2010 / Accepted: 25 August 2010 /

Published online: 16 September 2010

(C) Springer Science+Business Media, LLC 2010

\begin{abstract}
We show that if a line $\ell$ is an isolated line transversal to a finite family $\mathcal{F}$ of (possibly intersecting) balls in $\mathbb{R}^{3}$ and no two balls are externally tangent on $\ell$, then there is a subfamily $\mathcal{G} \subseteq \mathcal{F}$ of size at most 12 such that $\ell$ is an isolated line transversal to $\mathcal{G}$. We generalize this result to families of semialgebraic ovaloids.
\end{abstract}

Keywords Geometric transversals $\cdot$ Helly-type theorems $\cdot$ Line geometry $\cdot$ Ovaloids

\section{Introduction}

A straight line that intersects every member of a family $\mathcal{F}$ of convex compact subsets of $\mathbb{R}^{d}$ is called a line transversal to $\mathcal{F}$. A line transversal to a family $\mathcal{F}$ that cannot be moved without missing some member of $\mathcal{F}$ is said to be pinned by $\mathcal{F}$ (we also say that $\mathcal{F}$ is a pinning of that line). In other words, a line is pinned by $\mathcal{F}$ if it is an isolated point of the space of line transversals to $\mathcal{F}$.

A central question in geometric transversal theory is the study of sufficient conditions for the existence of a line transversal, and in particular of conditions that can be stated in the elegant form of a Helly-type theorem. One of the earliest examples is

Part of this work was achieved while the second author was staying at INRIA supported by the INRIA Internship Program.

X. Goaoc $\cdot$ S. Petitjean $(\bowtie)$

Project-team VEGAS, INRIA Nancy-LORIA, Vandœuvre, France

e-mail: Sylvain.Petitjean@inria.fr

X. Goaoc

e-mail: goaoc@loria.fr

S. König

Zentrum Mathematik, Technische Universität München, Garching bei München, Germany

e-mail: koenig@ma.tum.de 
the following theorem proven by Danzer [8] in 1957: If every 5 members in a finite family of disjoint unit disks have a line transversal, then the whole family has a line transversal. Danzer conjectured that this statement generalizes to families of disjoint unit balls in arbitrary dimension. This conjecture was recently settled in the positive by Cheong et al. [7], and one of the main ingredients in their proof is the following pinning theorem [7, Proposition 13]: If a finite family $\mathcal{F}$ of disjoint unit balls in $\mathbb{R}^{d}$ pins a line $\ell$, then some subset of $\mathcal{F}$ of size at most $2 d-1$ pins $\ell$. This pinning theorem can be understood as a Helly-type theorem for the existence of a line transversal locally near a pinned line: if no other line than the pinned line exists locally, this can be witnessed by $2 d-1$ of the balls.

Pinning theorems seem more "robust" than Helly-type theorems for the existence of a line transversal. For instance, Danzer's theorem is best possible in the sense that it becomes false if the disks are allowed to intersect or have arbitrary radii, whereas the pinning theorem remains valid for disjoint balls of arbitrary radii in $\mathbb{R}^{d}$ [5]. Similarly, polytopes in three dimensions have a pinning theorem under certain conditions [3] but admit no global Helly-type theorem [15].

In this paper, we show that the pinning theorem for disjoint balls also extends, in the three-dimensional case, to families of intersecting balls, provided no two balls are externally tangent on the line. More precisely, we prove the following.

Theorem 1 Let $\mathcal{F}$ be a finite family of balls in $\mathbb{R}^{3}$ that pin a line $\ell$. If no two balls are externally tangent in a point of $\ell$, then a subset of $\mathcal{F}$ of size at most 12 pins $\ell$.

While disjointedness of the objects is crucial for global Helly-type theorems, its relevance for the existence of a pinning theorem is not clear. On the one hand, whether disjointedness alone guarantees the existence of a pinning theorem for convex sets is a natural question, and we do not know of any minimal pinning of a line by more than 6 pairwise disjoint convex sets in $\mathbb{R}^{3}$ (see [7, Sect. 6]). On the other hand, Theorem 1 suggests that disjointedness may be relevant for pinning only insofar as it prevents certain singularities from occurring (a line tangent to two balls at their external tangency point is a singular point of the space of their line transversals, cf. the remark at the end of Sect. 3) and, in the polyhedral setting, such singularities can indeed lead to arbitrarily large minimal families of (intersecting) convex polytopes pinning a line [3, Theorem 3].

The proof of the pinning theorem for disjoint balls is based on properties of what are called cones of directions, which have been studied since Vincensini's original paper [18], that initiated geometric transversal theory. The cone of directions of a family of objects is the set of directions of its line transversals. The proof of the pinning theorem of Cheong et al. [7] is based on the observation that, for families of disjoint balls, this set is surprisingly well behaved [2, 5, 7, 14]: its connected components are strictly convex and are in one-to-one correspondence with the orders in which a line can intersect the family (the geometric permutations of the family). Interestingly, this approach fails to extend to situations where the balls intersect outside of the immediate vicinity of the pinned line: the cone of directions of a family of intersecting balls can be locally nonconvex at directions of transversals meeting the balls in distinct points (see, e.g., Fig. $2 d$ in Borcea et al. [5]). In some sense, the fact that the cone 
of directions be convex locally near a particular direction somehow requires that the balls be globally disjoint.

Our proof of Theorem 1, on the other hand, uses essentially local arguments and extends to solids bounded by ovaloids, a class of "locally sphere-like" surfaces (cf. Theorem 12). Let us sketch our proof briefly. It is well known that a family $\mathcal{F}$ pins a line $\ell$ if and only if the direction of $\ell$ is an isolated point of the cone of directions of $\mathcal{F}$ and that the cone of directions of $\mathcal{F}$ is the intersection of the cones of directions of the triples of balls in $\mathcal{F}$ (Lemma 3). We first prove that the cone of directions of three balls is "nice" in the sense that it is a manifold with boundary, and that this boundary is smooth except in directions of lines tangent to the three balls and passing through a point of tangency of two of the balls (Proposition 7). This allows us to recast the intersection of the $\left(\begin{array}{l}n \\ 3\end{array}\right)$ cones of triples as a sandwich region defined by semialgebraic functions in the plane, and Theorem 1 follows. We then give a geometric interpretation of the "first-order approximation" of the cone of directions at a smooth point (Lemma 10). A consequence of that interpretation is that our smoothness condition extends to ovaloids: the cone of directions of three ovaloids is smooth, except possibly at (points representing) directions of lines tangent to the three ovaloids and passing through a point of tangency of two of them (Lemma 11). The same argument on sandwich regions then yields a pinning theorem for ovaloids of "bounded description complexity" (Theorem 12).

The main idea leading to our interpretation of the "first-order approximation" of the cone of directions to three balls is to associate to a configuration of a ball and a line tangent to that ball a particular halfplane, which we call a screen; this construction was previously introduced by Cheong et al. [6] to analyze the stability of pinning configurations.

For a more general discussion of geometric transversal theory, we refer to the classic survey of Danzer et al. [9] and to the more recent ones of Eckhoff [10], Goodman et al. [12], and Wenger [19]. More specific discussions of recent progress on line transversals can be found in the survey of Holmsen [13] for the case of families of translated ovals in the plane and that of Goaoc [11] for the case of families of disjoint balls in arbitrary dimension.

Preliminaries and notation In what follows the space of directions in $\mathbb{R}^{3}$ is the sphere $\mathbb{S}^{2}$, and we assume that the lines are oriented. Given a direction $\mathbf{u}$, we denote by $\mathbf{u}^{\perp}$ the set of directions orthogonal to $\mathbf{u}$. In some cases it will be more convenient to identify opposite directions, and work in the real projective space $\mathbb{P}^{2}=\mathbb{P}^{2}(\mathbb{R})$ in view of the identification $\mathbb{P}^{2}=\mathbb{S}^{2} / \mathbb{Z}_{2}$.

Recall that a set is strictly convex if any supporting hyperplane intersects it in a single point and that a convex set is smooth if through any boundary point there is a unique supporting hyperplane. We say that two smooth surfaces are internally tangent (resp. externally tangent) at a point $\mathbf{p}$ if they are tangent at $\mathbf{p}$ and locally lie on the same side (resp. on opposite sides) of their common tangent plane.

Throughout the paper, we only consider closed balls with positive radius. We use the term family to denote a finite unordered set; in particular, in a family of balls we assume that the balls are pairwise distinct. We let $\left(\begin{array}{c}\mathcal{F} \\ k\end{array}\right)$ denote the set of all subsets of $\mathcal{F}$ of size $k$. 


\section{Cones of Directions}

Let $\mathcal{F}$ be a family of convex sets in $\mathbb{R}^{3}$. The directions of line transversals to $\mathcal{F}$ make up a subset $\mathcal{K}(\mathcal{F}) \subseteq \mathbb{S}^{2}$ called the cone of directions of $\mathcal{F}$. Here we extend properties of the cone of directions previously known for disjoint balls $[2,5,7,14]^{1}$ to arbitrary balls.

\subsection{Arbitrary Families of Balls}

We now assume we are given an arbitrary family of balls $\mathcal{F}$. The cone of directions of $\mathcal{F}$ can be seen as the image of the set of line transversals to $\mathcal{F}$ under the projection that maps a line to its direction. It is clear that the image of a connected component of transversals in this projection is connected. We can prove a stronger result.

Lemma 2 If $\mathcal{F}$ is a family of balls in $\mathbb{R}^{d}$, then there is a one-to-one correspondence between the connected components of line transversals to $\mathcal{F}$ and the connected components of $\mathcal{K}(\mathcal{F})$.

Proof Let $\phi$ be the function mapping a line to its direction. Let $\mathbf{u} \in \mathcal{K}(\mathcal{F})$, let $\Pi$ be a hyperplane of $\mathbb{R}^{d}$ with normal $\mathbf{u}$, and let $I$ denote the intersection of the orthogonal projections of the members of $\mathcal{F}$ on $\Pi$. Since each member of $\mathcal{F}$ is convex, $I$ is also convex and therefore connected. Since the line transversals to $\mathcal{F}$ with direction $\mathbf{u}$ are exactly the lines with direction $\mathbf{u}$ that intersect $I$, we get that $\phi^{-1}(\mathbf{u})$ is connected. Now, let $T_{1}, \ldots, T_{k}$ denote the connected components of line transversals to $\mathcal{F}$. Since $\phi$ is continuous, each $\phi\left(T_{i}\right)$ is connected. Since $\phi^{-1}(\mathbf{u})$ is connected for any $\mathbf{u} \in$ $\mathcal{K}(\mathcal{F})$, the $\phi\left(T_{i}\right)$ are pairwise disjoint. Since each of them is closed, it implies that each $\phi\left(T_{i}\right)$ is a connected component of $\mathcal{K}(\mathcal{F})$, and that each connected component of $\mathcal{K}(\mathcal{F})$ is the image of a single connected component of line transversals to $\mathcal{F}$.

We now describe various properties of the boundary of $\mathcal{K}(\mathcal{F})$. In what follows, a line is said to intersect a ball transversally if it intersects its interior. A line is an inner special bitangent if it is tangent to two elements of $\mathcal{F}$ and lies in a common tangent plane that separates them. In particular, two balls with intersecting interiors have no inner special bitangent, and the inner special bitangents to two externally tangent balls are the tangents through the point of tangency. A line is a tritangent if it is tangent to three elements of $\mathcal{F}$; a tritangent is called strict if it is not at the same time an inner special bitangent.

Lemma 3 Let $\mathcal{F}$ be a family of balls in $\mathbb{R}^{3}$. We have:

(i) $\mathcal{K}(\mathcal{F})=\bigcap_{X \in\left(\begin{array}{c}\mathcal{F} \\ 3\end{array}\right)} \mathcal{K}(X)$.

(ii) $\mathbf{u} \in \partial \mathcal{K}(\mathcal{F})$ only if the projections of the members of $\mathcal{F}$ on a plane orthogonal to $\mathbf{u}$ intersect with empty interior.

\footnotetext{
${ }^{1}$ Note that in these papers, which deal with disjoint objects, $\mathcal{K}(\mathcal{F})$ stands for the directions of lines piercing the sets of $\mathcal{F}$ in a specific order $\prec$. 
(iii) The boundary of $\mathcal{K}(\mathcal{F})$ consists of directions of tritangents and inner special bitangents.

(iv) $\mathcal{F}$ pins a line $\ell$ if and only if the direction of $\ell$ is an isolated point of $\mathcal{K}(\mathcal{F})$.

Proof Let $\mathbf{u}$ be a direction and let $\mathcal{F}_{\mathbf{u}}$ denote the family of the orthogonal projections of the elements of $\mathcal{F}$ on a plane orthogonal to a direction $\mathbf{u}$.

Helly's theorem in the plane yields that $\mathcal{F}_{\mathbf{u}}$ has nonempty intersection if and only if every triple has nonempty intersection. Thus, $\mathbf{u} \in \mathcal{K}(\mathcal{F})$ if and only if $\mathbf{u} \in \bigcap_{X \in\left(\begin{array}{c}\mathcal{F} \\ 3\end{array}\right)} \mathcal{K}(X)$, which proves statement (i).

If the intersection of the elements in $\mathcal{F}_{\mathbf{u}}$ has nonempty interior, then there exists a line that intersects every member of $\mathcal{F}$ transversally. Since any sufficiently small perturbation of that line remains a line transversal to $\mathcal{F}$, $\mathbf{u}$ is in the interior of $\mathcal{K}(\mathcal{F})$. This proves assertion (ii).

Let $\mathbf{u} \in \partial \mathcal{K}(\mathcal{F})$. Then there exists a triple $X \in\left(\begin{array}{c}\mathcal{F} \\ 3\end{array}\right)$ such that $\mathbf{u} \in \partial \mathcal{K}(X)$. By (ii), this implies that the orthogonal projections of the members of $X$ on a plane orthogonal to $\mathbf{u}$ intersect in a single point. This point is either on the boundary of the three projections or an external tangency point of two of them. In the former case $\mathbf{u}$ is the direction of a tritangent and in the latter the direction of an inner special bitangent. This implies statement (iii).

Assume that $\mathbf{u}$ is the only point of $\mathcal{K}(\mathcal{F})$ in some open set $R \subseteq \mathbb{P}^{2}$. Property (ii) implies that $\mathcal{F}$ has a unique line transversal $\ell$ with direction $\mathbf{u}$. Thus, $\ell$ is the only line transversal to $\mathcal{F}$ with direction in $R$. Since the set of all lines with direction in $R$ forms a neighborhood of $\ell$, it follows that $\mathcal{F}$ pins $\ell$. The reverse implication follows from Lemma 2.

Remark 1 The proofs of Lemmata 2 and 3 hold for general closed convex sets, with the understanding that (a) a line is "tangent" to a convex set if it intersects the set and lies in some supporting plane, and (b) a line intersects a convex set transversally if it intersects its relative interior but is not included in a plane containing the object. As we shall see in the next section, there are cases where the necessary condition (ii) of Lemma 3 is not sufficient.

\subsection{Arbitrary Triple of Balls}

Let us now turn our attention to a triple $T=\left\{B_{0}, B_{1}, B_{2}\right\}$ of possibly intersecting balls in $\mathbb{R}^{3}$. The main result of this section is Proposition 7 , which shows that $\mathcal{K}(T)$ has a "nice" structure. The proof is split across several lemmata:

- Lemma 4 characterizes what it means for a direction to be on the boundary of $\mathcal{K}(T)$;

- Lemma 5 describes the topology of $\mathcal{K}(T)$;

- and Lemma 6 shows that $\partial \mathcal{K}(T)$ is "almost always" smooth.

We first characterize the directions of transversals of $T$ appearing on the boundary of $\mathcal{K}(T)$ (extending [7, Lemma 9]) and those, among the directions of tritangents, that appear on the boundary of the cone of directions (extending [5, Proposition 3]). 
Lemma 4 The direction of a line transversal $\ell$ to $T$ belongs to $\partial \mathcal{K}(T)$ if and only if

(i) the three balls have no point in common,

(ii) $\ell$ is not tangent to two externally tangent balls at their tangency point while meeting the third ball in its interior, and

(iii) there is no other line transversal to $T$ parallel to $\ell$.

If $\ell$ is a (strict) tritangent, then condition (iii) can be replaced in the above equivalence by:

(iv) $\ell$ intersects the (interior of the) triangle formed by the balls' centers.

Proof Let $\mathbf{u}$ denote the direction of $\ell$. If the three balls have a point in common, then $\mathcal{K}(T)=\mathbb{S}^{2}$ has no boundary, so condition (i) is necessary. If $\ell$ is tangent to two externally tangent balls at their point $\mathbf{p}$ of tangency and meets the interior of the third ball, then any line through $\mathbf{p}$ with direction sufficiently close to $\mathbf{u}$ is a transversal to $T$, and $\mathbf{u} \in \mathcal{K}^{o}(T)$, where $\mathcal{K}^{o}(T)$ denotes the interior of $\mathcal{K}(T)$; condition (ii) is therefore necessary. If $T$ has another line transversal parallel to $\ell$, then the projections of the balls of $T$ along $\mathbf{u}$ intersect with nonempty interior, and Lemma 3(ii) ensures that $\mathbf{u} \in \mathcal{K}^{o}(T)$; condition (iii) is thus also necessary.

Before we show that the conditions are sufficient, let us first remark that for $\mathbf{v}$ close enough to $\mathbf{u}, T$ has a line transversal with direction $\mathbf{v}$ if and only if $T$ has a line transversal with direction $\mathbf{v}$ close to $\ell$. Indeed, let $\Pi_{\mathbf{v}}$ denote the plane through the origin with normal $\mathbf{v}$. The set of transversals to $T$ with direction $\mathbf{v}$ are precisely those that meet $\Pi_{\mathbf{v}}$ in a point of the intersection of the orthogonal projections of the balls on $\Pi_{\mathbf{v}}$. Since the orthogonal projection of a fixed ball on $\Pi_{\mathbf{v}}$ depends continuously on $\mathbf{v}$, it follows that for $\mathbf{v}$ close enough to $\mathbf{u}, T$ has a line transversal with direction $\mathbf{v}$ if and only if $T$ has a line transversal with direction $\mathbf{v}$ close to $\ell$.

Now, assume that (i), (ii), and (iii) hold. First consider the case where $\ell$ is not tritangent to $T$. Then, by Lemma 3(iii) $\ell$ is an inner special bitangent to two of the balls and condition (ii) implies that these balls meet $\ell$ in distinct points, and are thus disjoint. The perturbation argument used in [7, Lemma 9] guarantees that there are directions $\mathbf{v}$ arbitrarily close to $\mathbf{u}$ such that these two balls, and therefore $T$, have no line transversal and therefore that $\mathbf{u} \in \partial \mathcal{K}(T)$. In the case where $\ell$ is not tritangent, (i), (ii), and (iii) are thus sufficient.

Consider now the case where $\ell$ is tritangent to $T$. If the balls meet $\ell$ in distinct points, then, again, the same perturbation argument [7, Lemma 9] guarantees that $\mathbf{u} \in \partial \mathcal{K}(T)$. Condition (i) requires that at least two tangency points be distinct, so it remains to consider the case where two balls, say $B_{0}$ and $B_{1}$, are tangent to $\ell$ at the same point and the third ball is tangent at a different point. If $B_{0}$ and $B_{1}$ are externally tangent, then there is a direction $\mathbf{v}$ in the plane of tangency of $B_{0}$ and $B_{1}$ and arbitrarily close to $\mathbf{u}$ such that the line with direction $\mathbf{v}$ passing through the point of tangency of $B_{0}$ and $B_{1}$ misses $B_{2}$; clearly, $T$ has no transversal with such a direction $\mathbf{v}$, and $\mathbf{u} \in \partial \mathcal{K}(T)$. If $B_{0}$ and $B_{1}$ intersect properly, their bounding spheres intersect in a circle $\Lambda$. Observe that $\ell$ is tangent to $\Lambda$ in its plane and that there is a direction $\mathbf{v}$ in this plane arbitrarily close to $\mathbf{u}$ such that the tangent to $\Lambda$ with direction $\mathbf{v}$ close to $\ell$ misses $B_{2}$; clearly, $T$ has no transversal with such a direction $\mathbf{v}$, 

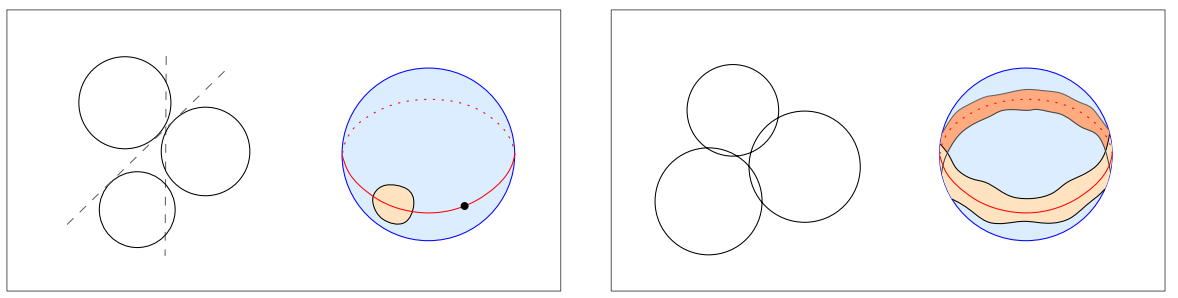

Fig. 1 Possible topologies of the cone of directions of three balls: (left) contractible connected components, possibly reduced to a point; (right) a strip containing the $\mathbb{S}^{1}$ of directions in the plane of centers in its interior. Note that, in all cases, the figure is symmetric with respect to the $\mathbb{S}^{1}$ of directions in the plane of centers

Fig. 2 Retracting $\mathcal{K}(T)$ onto $\mathcal{K}\left(T^{\prime}\right)$

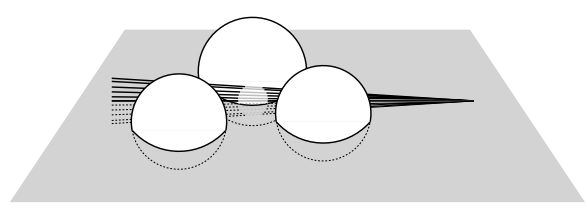

and $\mathbf{u} \in \partial \mathcal{K}(T)$. This proves that in the case where $\ell$ is tritangent, conditions (i), (ii), and (iii) are also sufficient.

Property (iv) was observed for tritangents to triples of disjoint balls by Borcea et al. [5, Proposition 3]. Their proof easily extends to intersecting balls.

Next, we describe the topology of the cone of directions (cf. Fig. 1), extending [5, Proposition 4].

Lemma 5 Let $C$ be a connected component of $\mathcal{K}(T)$. The following holds:

(i) $C$ is a single point if and only if there is a line with that direction that is pinned by $T$.

(ii) $C$ is all of $\mathbb{S}^{2}$ if and only if the three balls have a point in common.

(iii) $C$ is a strip that contains the $\mathbb{S}^{1}$ of directions of the plane of centers in its interior if and only if the balls in $T$ intersect pairwise but not triplewise. In that case, $C$ is the only connected component of $\mathcal{K}(T)$.

(iv) In all other cases, $C$ is contractible and is the closure of its interior.

Proof Let $\Pi$ denote the plane containing the centers of the balls in $T$ (or any such plane, if the centers are aligned). Statement (i) follows from Lemma 3(iv). If there is a point common to the three balls in $T$, then $\mathcal{K}(T)=\mathbb{S}^{2}$. If the intersection of the three balls is empty, then $T$ has no line transversal with direction orthogonal to $\Pi$, and $\mathcal{K}(T) \neq \mathbb{S}^{2}$. This proves statement (ii).

All the lines between a line transversal $\ell$ to $T$ and its mirror image with respect to $\Pi$ are also line transversals to $T$, as observed by Borcea et al. [5, Proposition 4] (see Fig. 2). When the three balls have no point in common, $T$ has no line transversal orthogonal to $\Pi$, and the set of line transversals to $T$ can be retracted onto the set of line transversals to $T$ contained in $\Pi$. This induces a retraction from $\mathcal{K}(T)$ onto the cone of directions of the disks $T^{\prime}=\left\{B_{i} \cap \Pi \mid i=0, \ldots, 2\right\}$. 
Now on to statement (iii). Observe that $\mathcal{K}\left(T^{\prime}\right)=\mathbb{S}^{1}$ if and only if the disks, and hence the balls, intersect pairwise. One direction follows from Helly's theorem in one dimension, the other from the observation that if two disks are disjoint, then $T^{\prime}$ has no transversal in the direction orthogonal to the vector joining their centers.

We now show that, assuming every two balls in $T$ intersect but the three balls have empty intersection, $\mathcal{K}(T)$ is a strip containing $\mathcal{K}\left(T^{\prime}\right)=\mathbb{S}^{1}$ in its interior. Observe first that under those assumptions no direction of $\partial \mathcal{K}(T)$ is parallel to $\Pi$. Indeed, assume for a contradiction that $\mathbf{u}$ is such a direction and let $\ell$ be the (unique by Lemma 4 ) line transversal to $T$ with direction $\mathbf{u}$. If $\ell$ is an inner special bitangent to two balls, these balls must be tangent and $\ell$ must meet them in their point of tangency; the interior of the third ball must intersect $\ell$ (otherwise, as $\ell$ is in the plane of centers, the third ball would not intersect one of the first two), and $\mathbf{u} \notin \partial \mathcal{K}(T)$ by Lemma 4 . If $\ell$ is not an inner special bitangent to any pair in $T$, then it is tangent to all three balls; in the plane $\Pi$, the three disks must be on the same side of $\ell$, and $\mathbf{u}$ is therefore clearly not on $\partial \mathcal{K}(T)$.

Now, if every pair in $T$ intersects with the three balls having empty intersection, then we can retract $\mathcal{K}(T)$ onto the $\mathbb{S}^{1}$ of directions parallel to $\Pi$, and no direction from this $\mathbb{S}^{1}$ lies in $\partial \mathcal{K}(T) ; \mathcal{K}(T)$ is thus a strip that contains the $\mathbb{S}^{1}$ of directions of the plane of centers in its interior. Conversely, if $\mathcal{K}(T)$ has this geometry, then the cone of directions of $T^{\prime}$ is an $\mathbb{S}^{1}$ (since the projection of any line transversal to $T$ onto $\Pi$ gives a line transversal to $T^{\prime}$ ). It follows that the disks in $T^{\prime}$ intersect pairwise but not triplewise, and so do the balls in $T$, proving (iii).

Finally, in all other cases a connected component of $\mathcal{K}\left(T^{\prime}\right)$ is an interval, and (iv) follows.

The cone of directions of a triple of disjoint balls is strictly convex, but this property fails for intersecting balls [5]. However, we can still show that the boundary of the cone is "almost always" smooth.

Lemma 6 A direction $\mathbf{u}$ is a singular point of $\partial \mathcal{K}(T)$ if and only if the intersection of the three balls is empty and there exists a line with direction $\mathbf{u}$ that is:

(i) pinned by the three balls, or

(ii) tangent to all three balls, meeting two of them in the same point in which they are externally tangent.

Proof Let $\mathbf{u}$ be a point of $\partial \mathcal{K}(T)$. By Lemma 3(ii) there exists a unique line transversal, say $\ell$, to the three balls having direction $\mathbf{u}$. We argue that if $\mathbf{u}$ is a singular point, then $\ell$ must satisfy condition (i) or (ii).

By Lemma 3(iii), the boundary of $\mathcal{K}(T)$ consists of two types of arcs, arcs of directions of inner special bitangents to some pair $\left\{B_{i}, B_{j}\right\}$ and arcs of directions of tritangents to $\left\{B_{0}, B_{1}, B_{2}\right\}$. The directions of inner special bitangents to two distinct balls is either empty or a smooth conic (cf. [5]). Note that Lemma 3(ii) implies that, for directions on the boundary of $\mathcal{K}(T)$, two such arcs meet in a tritangent direction. Therefore, if $\mathbf{u}$ is a singularity of $\partial \mathcal{K}(T)$, it must be the direction of a tritangent. 

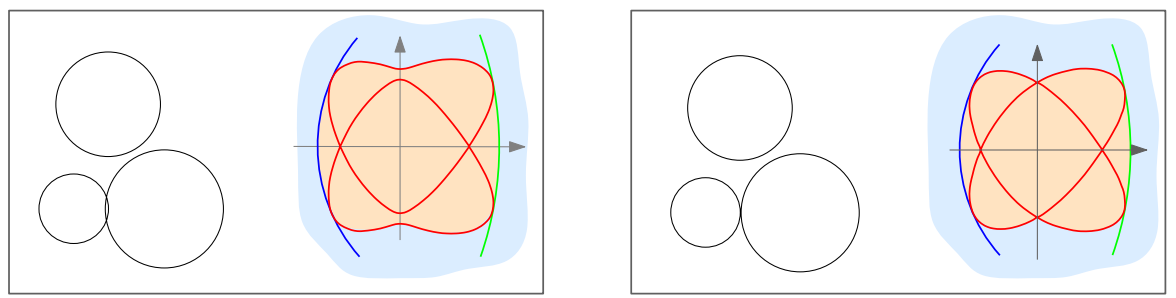

Fig. 3 (Color online) Two triples of balls (represented by their trace on their plane of centers) and a planar depiction of their cone of directions. The direction-sextic is drawn in red, conics of directions of inner special bitangents are drawn in blue and green; the orange region is the cone of directions (a connected set in this example), and the horizontal axis corresponds to directions in the plane of the balls' centers. Observe that when the balls intersect properly (left), the cone of directions is smooth as when the balls are disjoint; whereas, when some of the balls are tangent (right), the cone of directions exhibits a singularity

Let $\mathbf{c}_{i}$ and $s_{i}$ denote, respectively, the center and squared radius of $B_{i}$. The directions of tritangents to $\left\{B_{0}, B_{1}, B_{2}\right\}$ make up an algebraic curve of degree 6 in $\mathbb{P}^{2}$, the direction-sextic $\sigma_{B_{0} B_{1} B_{2}}(\mathbf{u})$ of these three balls. Letting $\mathbf{e}_{i j}=\mathbf{c}_{j}-\mathbf{c}_{i}$ and $\delta_{i j}=\left\langle\mathbf{e}_{i j}, \mathbf{e}_{i j}\right\rangle$ and writing, for a given direction $\mathbf{u} \in \mathbb{P}^{2}$,

$$
q=q(\mathbf{u})=\langle\mathbf{u}, \mathbf{u}\rangle \quad \text { and } \quad t_{i j}=t_{j i}=\left\langle\mathbf{e}_{i j} \times \mathbf{u}, \mathbf{e}_{i j} \times \mathbf{u}\right\rangle=\delta_{i j} q-\left\langle\mathbf{e}_{i j}, \mathbf{u}\right\rangle^{2},
$$

the direction-sextic of $B_{0}, B_{1}, B_{2}$ rewrites as a Cayley determinant [5, Proposition 2]:

$$
\sigma_{B_{0} B_{1} B_{2}}(\mathbf{u})=\operatorname{det}\left(\begin{array}{ccccc}
0 & 1 & 1 & 1 & 1 \\
1 & 0 & q s_{0} & q s_{1} & q s_{2} \\
1 & q s_{0} & 0 & t_{01} & t_{02} \\
1 & q s_{1} & t_{01} & 0 & t_{12} \\
1 & q s_{2} & t_{02} & t_{12} & 0
\end{array}\right)=0 .
$$

Figure 3 illustrates typical situations.

We now assume that $\mathbf{u}$ lies on the boundary of $\mathcal{K}(T)$ and is a singular point of the direction-sextic, i.e., that the gradient of $\sigma_{B_{0} B_{1} B_{2}}$ in $\mathbf{u}$ vanishes. To analyze the gradient of the direction-sextic in a direction on $\partial \mathcal{K}(T)$, we proceed as in Borcea et al. [5]. Consider the projection along a direction $\mathbf{u}$ of the three balls and a tritangent $\ell$ with that direction; by Lemma $4, \mathbf{u}$ is on the boundary of the cone of directions if and only if the projection of $\ell$ lies in the projection $\Delta$ of the triangle of centers. We equip $\mathbb{R}^{3}$ with a coordinate frame with axes $x, y, z$ such that this projected triangle lies in the plane $z=0$ (i.e., we consider tritangent lines having $\mathbf{e}_{3}=(0,0,1)$ as direction) and has its vertices at $\tilde{\mathbf{c}}_{0}=(0,0,0), \tilde{\mathbf{c}}_{1}=(a, 0,0), \tilde{\mathbf{c}}_{2}=(b, c, 0)$, with the understanding that there is a point $\mathbf{p}$ inside,

$$
\mathbf{p}=\sum p_{i} \tilde{\mathbf{c}}_{i}, \quad \text { with } p_{0}, p_{1}, p_{2} \geq 0, \sum p_{i}=1 .
$$

Note that the squared distance from $\mathbf{p}$ to vertex $\tilde{\mathbf{c}}_{i}$ is the squared radius of $B_{i}$, i.e., $s_{i}=\left\langle\mathbf{p}-\tilde{\mathbf{c}}_{i}, \mathbf{p}-\tilde{\mathbf{c}}_{i}\right\rangle$. Then, we use three real parameters $x_{0}, x_{1}, x_{2}$ to describe the possible positions of the three centers of $B_{0}, B_{1}, B_{2}$ :

$$
\mathbf{c}_{0}=\tilde{\mathbf{c}}_{0}+x_{0} \mathbf{e}_{3}, \quad \mathbf{c}_{1}=\tilde{\mathbf{c}}_{1}+x_{1} \mathbf{e}_{3}, \quad \mathbf{c}_{2}=\tilde{\mathbf{c}}_{2}+x_{2} \mathbf{e}_{3},
$$


where we assume w.l.o.g. that $x_{2} \geq x_{1} \geq x_{0}$. Substituting in (1) we can express the direction-sextic $\sigma_{B_{0} B_{1} B_{2}}$ and its derivatives in the direction $\mathbf{e}_{3}=(0,0,1)$ as a function of $x_{0}, x_{1}, x_{2}$ depending on the parameters $a, b, c, p_{0}, p_{1}, p_{2}$. Denoting $\mathbf{u}=\left(u_{0}, u_{1}, u_{2}\right)$, the computation gives:

$$
\begin{aligned}
\left(\frac{\partial \sigma}{\partial u_{0}}\right)(0,0,1)= & 16 a^{2} c^{2}\left[a p_{1}\left(x_{1} p_{0}-x_{0} p_{0}-x_{2} p_{2}+x_{1} p_{2}\right)\right. \\
& \left.-b p_{2}\left(-x_{2} p_{0}-x_{2} p_{1}+x_{0} p_{0}+x_{1} p_{1}\right)\right] \\
\left(\frac{\partial \sigma}{\partial u_{1}}\right)(0,0,1)= & -16 a^{2} c^{3} p_{2}\left[p_{0}\left(x_{0}-x_{2}\right)+p_{1}\left(x_{1}-x_{2}\right)\right] \\
\left(\frac{\partial \sigma}{\partial u_{2}}\right)(0,0,1)= & 0 .
\end{aligned}
$$

Note that the partial derivatives all vanish if $a=0$ or $c=0$. If $a=0$ then the centers of two balls coincide and these balls must have equal radii to allow for a common tangent, a situation our assumptions rule out. If $c=0$ then the three centers are aligned; by symmetry of revolution around the line of the centers, the cone of directions is a circle, and therefore smooth. We can also rule this case out, and assume from now on that $a c \neq 0$.

First assume $\Delta$ is degenerate, i.e., it collapses to a segment $s$. Then $\ell$ is a tritangent line contained in a plane tangent to all three balls and is also contained in the plane of centers $P$. Since $\ell$ hits $s$ by Lemma 5(iv), those centers must lie on both sides of $\ell$ in $P$, say $\mathbf{c}_{i}$ on one side and $\mathbf{c}_{j}, \mathbf{c}_{k}$ on the other. Since there are no strict tritangent directions, either $\mathbf{u}$ is isolated in $\mathcal{K}(T)$, in which case the three balls pin $\ell$ and we are in case (i); or $\mathbf{u}$ is not isolated, in which case $\ell$ is the intersection of the sets of inner special bitangents to $\left\{B_{i}, B_{j}\right\}$ and $\left\{B_{i}, B_{k}\right\}$. These two sets correspond, in the space of directions, to two conics, one being internally tangent to the other at $\mathbf{u}$. In other words, $\mathcal{K}(T)$ is not singular at $\mathbf{u}$.

Now assume $\Delta$ is nondegenerate. Suppose $\mathbf{u}$ is a strict tritangent direction, i.e., it is a singular point of $\sigma$ and point $\mathbf{p}$ is strictly inside $\Delta$ by Lemma 5(iv). This implies that the three derivatives above vanish and $p_{i}>0, i=0,1,2$. From $\left(\frac{\partial \sigma}{\partial u_{1}}\right)(0,0,1)=0$ we conclude that $x_{0}=x_{1}=x_{2}$. Geometrically, this means that the three balls intersect in a common point and every line through this point is a transversal to the three balls. Therefore, $\mathcal{K}(T)=\mathbb{P}^{2}$ which has no singular point, a contradiction.

Thus, $\mathbf{u}$ must also be a direction of inner special bitangent and exactly one $p_{i}$ is zero (if two of them vanish, $\mathbf{p}$ is a vertex of $\Delta$, i.e., $\ell$ goes through the center of one ball, implying that this ball has radius 0 , which we rule out). Let $j$ and $k$ denote the other two indices; notice then that $\ell$ is an inner special bitangent to $B_{j}$ and $B_{k}$. We then obtain from the vanishing of the partial derivatives above that $x_{j}=x_{k}$. Thus, $B_{j}$ and $B_{k}$ meet $\ell$ in the same point, and are externally tangent at that point; we are then in case (ii).

Altogether, we have that if $\mathbf{u}$ is a singularity, then $\ell$ satisfies condition (i) or (ii). Conversely, if $\ell$ satisfies (i), then $\mathcal{K}(T)$ is, locally around $\mathbf{u}$, reduced to a point and $\mathbf{u}$ is a singularity. If $\ell$ satisfies (ii), then the gradient of $\sigma$ in $\mathbf{u}$ vanishes and $\mathbf{u}$ is therefore a singularity. 
As a consequence, we obtain that the cone of directions of three balls has a nice structure.

Proposition 7 If $T$ is a triple of balls, no two externally tangent, then every connected component of $\mathcal{K}(T)$ is either a single point, $\mathbb{S}^{2}$ or a semialgebraic 2-manifold with a smooth boundary.

Proof As observed in [1], the set of line transversals to a family of semialgebraic objects of "bounded description complexity" (i.e., semialgebraic sets defined using a bounded number of polynomial equalities and inequalities of bounded degrees), seen as a 4-dimensional subset of line space, is a semialgebraic set. For $T$ a triple of balls, the set of transversals to $T$ is therefore a semialgebraic set, and so is its projection $\mathcal{K}(T)$ onto the space of directions. The fact that each connected component is a single point or a 2-manifold with boundary (except when $\mathcal{K}(T)=\mathbb{S}^{2}$ ) follows from Lemma 5. The fact that the boundary is smooth when $\mathcal{K}(T)$ is not reduced to a single point and no two balls are externally tangent follows from Lemma 6.

\section{Pinning Theorem for Intersecting Balls}

We can now prove Theorem 1, which states that if $n$ balls in $\mathbb{R}^{3}$ pin a line and no two balls are externally tangent on the line, then a subset of at most 12 of these balls pins that line.

Proof of Theorem 1 Let $\mathcal{F}$ be a finite family of balls in $\mathbb{R}^{3}$ that pins a line $\ell$, no two balls being externally tangent on $\ell$. Assume no triple of balls of $\mathcal{F}$ already pins $\ell$, as otherwise the statement is trivially true. Let $\mathbf{u}$ denote the direction of $\ell$. By Lemma 3(iv), $\mathbf{u}$ is an isolated point of $\mathcal{K}(\mathcal{F})$, and it suffices to find a subfamily $Y \subseteq \mathcal{F}$ of size at most 12 such that $\mathbf{u}$ is an isolated point of $\mathcal{K}(Y)$ to prove the statement.

By Lemma 3(i), $\mathcal{K}(\mathcal{F})$ is the intersection of the cones $\mathcal{K}(T)$ for all triples $T \subset \mathcal{F}$ and dropping any triple $T$ such that $\mathbf{u} \in \mathcal{K}^{o}(T)$ keeps $\mathbf{u}$ isolated in the intersection. Thus, if we denote by $\mathcal{N}$ the set of triples $T \subset \mathcal{F}$ such that $\mathbf{u}$ is on the boundary of $\mathcal{K}(T)$, we have that $\mathbf{u}$ is an isolated point of $\bigcap_{T \in \mathcal{N}} \mathcal{K}(T)$.

By Proposition 7, for every $T \in \mathcal{N}$ there exists an arbitrarily small neighborhood $U_{T}$ of $\mathbf{u}$ such that $\mathcal{K}(T) \cap U$ is homeomorphic to a halfplane. Let $U$ denote a neighborhood of $\mathbf{u}$ such that $U \cap \mathcal{K}(T)$ is homeomorphic to a halfplane for all $T \in \mathcal{N}$. Let $\eta_{T}$ denote the normal to $\partial \mathcal{K}(T)$ in $\mathbf{u}$ that points outward of $\mathcal{K}(T)$, and consider an orthogonal coordinate system $(\mathbf{u}, x, y)$ in $U$ such that $\left\langle\eta_{T}, y\right\rangle \neq 0$ for all $T \in \mathcal{N}$. We split $\mathcal{N}$ into two subsets:

$$
\mathcal{N}^{+}=\left\{T \in \mathcal{N} \mid\left\langle\eta_{T}, y\right\rangle>0\right\} \quad \text { and } \quad \mathcal{N}^{-}=\left\{T \in \mathcal{N} \mid\left\langle\eta_{T}, y\right\rangle<0\right\} .
$$

By the semialgebraic implicit function theorem [4, p. 97], for $U$ small enough $\partial \mathcal{K}(T)$ can be written in the form $y=f_{T}(x)$, where $f_{T}$ is a semialgebraic function. Since $\mathcal{K}(T) \cap U$ is homeomorphic to a halfplane, it follows that in $U, \mathcal{K}(T)$ can be written as $\left\{(x, y) \mid y \leq f_{T}(x)\right\}$ if $T \in \mathcal{N}^{+}$and as $\left\{(x, y) \mid y \geq f_{T}(x)\right\}$ if $T \in \mathcal{N}^{-}$. 
Now, observe that in $U, \bigcap_{T \in \mathcal{N}} \mathcal{K}(T)$ is exactly the set of points that are below all curves in $\left\{f_{T} \mid T \in \mathcal{N}^{+}\right\}$and above all curves in $\left\{f_{T} \mid T \in \mathcal{N}^{-}\right\}$. Since the functions $f_{T}$ are semialgebraic, near $\mathbf{u}$ they either coincide or there is a neighborhood of $\mathbf{u}$ in which they only meet in $\mathbf{u}$. It follows that there exists $\epsilon>0$ and four subsets $A, B \in$ $\mathcal{N}^{+}$and $C, D \in \mathcal{N}^{-}$such that on the interval $[-\epsilon, 0]$, all functions in $\left\{f_{T} \mid T \in \mathcal{N}^{+}\right\}$ are above $f_{A}$ and all functions in $\left\{f_{T} \mid T \in \mathcal{N}^{-}\right\}$are below $f_{C}$, and similarly on $[0, \epsilon]$ all functions in $\left\{f_{T} \mid T \in \mathcal{N}^{+}\right\}$are above $f_{B}$ and all functions in $\left\{f_{T} \mid T \in \mathcal{N}^{-}\right\}$are below $f_{D}$. As a consequence,

$$
\mathcal{K}(A) \cap \mathcal{K}(B) \cap \mathcal{K}(C) \cap \mathcal{K}(D) \cap U=\{\mathbf{u}\}
$$

and $A \cup B \cup C \cup D$ is a subset of $\mathcal{F}$ of size at most 12 that pins $\ell$.

Remark 2 It is not clear to us whether the condition that no two balls be externally tangent on the line is really needed for the pinning theorem to hold. However, we do note that these configurations are indeed particular, in the sense that the space of oriented line transversals to two externally tangent balls is singular at any line through their tangency point. Indeed, the set of lines through the tangency point is 2 dimensional, and removing that set from the set of lines intersecting the two balls creates two connected components, each being 4 dimensional.

Remark 3 Extending the proof of Theorem 1 to pinnings in higher dimension seems difficult. First, generalizing Lemma 3(i), one would have to work with cones of directions of $d$ balls in $\mathbb{R}^{d}$, and identifying the singularities of such cones may not be an easy task. Second, and more importantly, our proof exploits the fact that, in the plane, a lower/upper envelope of semialgebraic functions is defined, near one of its vertices, by a constant number of the functions ( 2 in this case). Already in dimension 3 this is not true for general semialgebraic sets (consider the lower envelope of several copies of the paraboloid $z=x^{2}+2 y^{2}$, rotated around the $z$ axis, in the neighborhood of $(0,0,0))$, and it is not clear why it would be true for cones of directions.

\section{Extension to Ovaloids}

An ovaloid is a smooth closed surface in $\mathbb{R}^{3}$ with strictly positive Gauss curvature everywhere. According to a classical theorem of Hadamard (1857), ovaloids are topologically spheres (cf. [16, Chaps. 4 \& 6]). More precisely, Hadamard's theorem asserts that an ovaloid is the boundary of a bounded open strictly convex set. By abuse of language, we use the term ovaloid both for the surface and for the bounded solid it encloses.

In this section, we extend our pinning theorems to families of ovaloids of bounded description complexity. A key idea is to represent a first-order approximation of the cone of directions of three balls in a given direction as the cone of directions of three well-chosen parallel halfplanes. Our proof is split across several lemmata:

- Lemma 8 extends our characterization of the boundary of the cone of directions from triples of balls to triples of ovaloids; 
- Lemma 9 describes the cone of directions of three parallel halfplanes;

- Lemma 10 shows that the first-order approximation of the cone of directions of three balls in a neighborhood of a given boundary direction can be represented by the cone of directions of three well-chosen halfplanes;

- Lemma 11 then extends our sufficient condition for the smoothness of the cone of directions to triples of ovaloids, and our pinning theorem for ovaloids (Theorem 12) follows.

\subsection{Boundary of the Cone of Directions of Three Ovaloids}

An ovaloid has positive Gauss curvature everywhere. Its second fundamental form relative to the inward normal is positive definite at every point. It follows that its principal curvatures $\kappa_{1}$ and $\kappa_{2}$ are strictly positive everywhere. Assume $\kappa_{1}$ is the largest of the two principal curvatures. As is well known, any ball internally tangent to a smooth convex surface at $\mathbf{p}$ of radius less than the principal radius of curvature $1 / \kappa_{1}(\mathbf{p})$ is inside the surface locally around $\mathbf{p}$. Actually, picking a radius "small enough" will ensure that the ball is not just locally but globally inside the ovaloid. ${ }^{2}$ Similarly, any ball internally tangent at $\mathbf{p}$ of radius more than the principal radius of curvature $1 / \kappa_{2}(\mathbf{p})$ is outside the surface locally around $\mathbf{p}$, and picking a radius large enough will ensure that the ovaloid is globally inside the ball. One can therefore "sandwich" an ovaloid at any of its points between two balls. Note that this is not true for all smooth strictly convex sets: for instance, the set defined by $f \leq 0$ where $f=x^{4}+y^{4}+z^{4}-1$ is smooth strictly convex, but the two principal curvatures of the zero-set of $f$ at all extreme points along the $x, y$, and $z$ axes vanish.

This "sandwich" property allows us to extend the characterization of the boundary of the cone of directions of Lemma 4 to ovaloids.

Lemma 8 Let $T$ be a triple of ovaloids. The direction of a line transversal $\ell$ to $T$ belongs to $\partial \mathcal{K}(T)$ if and only if

(i) the three ovaloids have no point in common,

(ii) $\ell$ is not tangent to two externally tangent ovaloids at their tangency point while meeting the third ovaloid in its interior, and

(iii) there is no other line transversal to $T$ parallel to $\ell$.

Proof The three conditions are clearly necessary, so we prove the converse. Let $\mathbf{u}$ denote the direction of $\ell$. By (iii), the orthogonal projections of the ovaloids on a plane orthogonal to $\mathbf{u}$ intersect in a single point, so $\ell$ is either an inner special bitangent or a tritangent. In the former case, the same argument as in Lemma 4 shows that $\mathbf{u} \in \partial \mathcal{K}(T)$ if (i) and (iii) hold. In the latter case, let $T^{+}$denote a triple of balls, where each ball is tangent to an ovaloid of $T$ at its tangency point with $\ell$ and contains that ovaloid. Now, observe that $\mathbf{u} \in \partial \mathcal{K}\left(T^{+}\right)$by Lemma 4 and that $\mathcal{K}(T) \subseteq \mathcal{K}\left(T^{+}\right)$. It follows that $\mathbf{u}$ is in the boundary of $\mathcal{K}(T)$, and the statement follows.

\footnotetext{
${ }^{2}$ We do not need to be more precise here, but note that, by Blaschke's Rolling Theorem (cf. [17]), any ball of radius less than the infimum of $1 / \kappa_{1}(\mathbf{p})$ can roll along the surface of the ovaloid while always staying inside.
} 


\subsection{Screens}

We associate to a pair $(\ell, B)$ of a line $\ell$ intersecting a ball $B$ an object, which we call a screen, as follows. If $\ell$ meets the interior of $B$, then the screen of $(\ell, B)$ is the plane orthogonal to $\ell$ that passes through the center of $B$. If $\ell$ is tangent to $B$, we let $\mathbf{t}$ denote that tangency point and $\mathbf{v}$ the outward normal to $B$ at $\mathbf{t}$, and define the screen of $(\ell, B)$ as the (closed) halfplane

$$
\{\mathbf{x} \mid\langle\mathbf{t x}, \mathbf{u}\rangle=0 \text { and }\langle\mathbf{t x}, \mathbf{v}\rangle \leq 0\},
$$

that is, the intersection of the plane perpendicular to $\ell$ through $\mathbf{t}$ with the (closed) halfspace "tangent" to $B$ at $\mathbf{t}$ and containing $B$. When a screen is a halfplane, we call its boundary line in its affine hull its boundary.

To simplify the presentation, we assume here that a line parallel to a halfplane intersects it at infinity (and thus $\mathbf{u}^{\perp}$ is in the cone of directions of any triple of screens lying in planes orthogonal to $\mathbf{u}$ ). Note that this convention has no effect on pinning problems.

Lemma 9 Let $T$ be a triple of balls, $\ell$ a line transversal to $T$ with direction $\mathbf{u}$, and $S$ the triple of screens defined by $\ell$ and the balls of $T$. Let $\Gamma$ denote the great circle of directions of transversals to the boundaries of the screens in S. The following holds:

(i) If $T$ pins $\ell$, then $\mathcal{K}(S)=\Gamma$.

(ii) If $\mathbf{u} \in \partial \mathcal{K}(T)$ and $T$ does not pin $\ell$, then $\mathcal{K}(S)$ is the union of $A \cap B$ and its symmetric, where $A$ and $B$ are closed hemispheres bounded by $\Gamma$ and $\mathbf{u}^{\perp}$ respectively.

(iii) If $\mathbf{u} \in \mathcal{K}^{o}(T)$, then $\mathcal{K}(S)=\mathbb{S}^{2}$.

Proof If $T$ pins $\ell$, then $T$ consists of three balls tangent to $\ell$ with a common tangent plane $\Pi$ and whose positions with respect to $\Pi$ alternate. Thus, the screens of $S$ are bounded by lines contained in $\Pi$ and their positions with respect to $\Pi$ also alternate. Since the set of directions contained in $\Pi$ is exactly $\Gamma$, statement (i) follows.

We now assume that $T$ does not pin $\ell$ and that $\mathbf{u} \in \partial \mathcal{K}(T)$. By Lemma 3(iii), $\ell$ is an inner special bitangent to two of the balls or is tangent to all three balls in $T$. Let $S_{1}, S_{2}$, and $S_{3}$ be the three screens in $S$.

We first consider the case where $\ell$ is an inner special bitangent to the first two balls. Then, the boundaries of $S_{1}$ and $S_{2}$ are parallel and span a plane $\Pi$ that contains $\ell$. Moreover, $\Pi$ separates $S_{1}$ and $S_{2}$. Since $T$ does not pin $\ell, S_{3}$ is a plane, a halfplane whose boundary intersects $\Pi$ in a single point, or a halfplane whose boundary is contained in $\Pi$ and that lies on the same side as $S_{2}$ with respect to $\Pi$. In each of these cases, it can easily be checked that $\mathcal{K}(S)=\mathcal{K}\left(\left\{S_{1}, S_{2}\right\}\right)$. Consider a direction $\mathbf{v}$. If a direction $\mathbf{v}$ is orthogonal to $\mathbf{u}$, then by our convention $\mathbf{v}$ is in $\mathcal{K}(S)$. If $\mathbf{v}$ makes a positive dot product with $\mathbf{u}$, then $\mathbf{v} \in \mathcal{K}\left(\left\{S_{1}, S_{2}\right\}\right)$ if and only if $\mathbf{v}$ is parallel to $\Pi$ or crosses it from the side of $S_{1}$ to that of $S_{2}$. If $\mathbf{v}$ makes a negative dot product with $\mathbf{u}$, then we are in the symmetric case, and the result follows. The case where $\ell$ is an inner special bitangent to another pair is handled similarly.

Now, assume that $\ell$ is tangent to all three balls in $T$ but is not an inner special bitangent to any two of them. In particular, this implies that no two screens in $S$ have 
parallel boundaries. The orthogonal projections of the $S_{i}$ 's on a plane orthogonal to $\mathbf{u}$ intersect in a single point. Now, if we consider a direction $\mathbf{v}$ moving on $\Gamma$ starting in $\mathbf{u}$, the orthogonal projections of the $S_{i}$ 's on a plane orthogonal to $\mathbf{v}$ change continuously, and the boundaries of these three halfplanes keep intersecting in a point. The intersection thus remains a single point unless two of the projected halfplanes become equal or opposite; this cannot happen, as it requires the boundaries of the corresponding screens to be parallel. Thus, the intersection of the projections of the screens in $S$ along any direction of $\Gamma$ is a single point. Conversely, the intersection of the projections of the screens in $S$ along any direction not in $\Gamma \cup \mathbf{u}^{\perp}$ is either empty or has nonempty interior. Thus, the boundary of $\mathcal{K}(S)$ consists of $\Gamma \cup \mathbf{u}^{\perp}$. A perturbation argument similar to [7, Lemma 9] shows that $\mathcal{K}(S)$ is, locally, on one side of $\Gamma$, and statement (ii) follows.

If $\mathbf{u} \in \mathcal{K}^{o}(T)$, then for any direction $\mathbf{v}$ the projections of the screens in $S$ along $\mathbf{v}$ intersect with nonempty interior. Statement (iii) follows.

\subsection{First-order Approximation of Cones of Directions}

The tangential cone $\mathcal{T}_{\mathbf{p}}(X)$ of a closed nonempty set $X \subset \mathbb{R}^{d}$ at a point $\mathbf{p}$ of its boundary is the set of all directions $\mathbf{d}$ such that $\mathbf{d}=\lim _{k \rightarrow \infty} \lambda_{k}\left(\mathbf{p}_{k}-\mathbf{p}\right)$, where $\lambda_{k}>0$, $\mathbf{p}_{k} \in X$ for each $k$ and $\mathbf{p}_{k} \rightarrow \mathbf{p}$. From the above definition, it is clear that $\mathbf{d}$ belongs to the tangential cone if there is a sequence $\left(\mathbf{p}_{k}\right)_{k \in \mathbb{N}}$ of points in $X$ converging to $\mathbf{p}$ such that the direction of the chords $\mathbf{p}_{k}-\mathbf{p}$ converges to $\mathbf{d}$. In particular, if $\partial X$ is smooth at $\mathbf{p}$, then $\mathcal{T}_{\mathbf{p}}(X)$ is a closed halfspace whose outward normal is the outward normal of $X$ at $\mathbf{p}$.

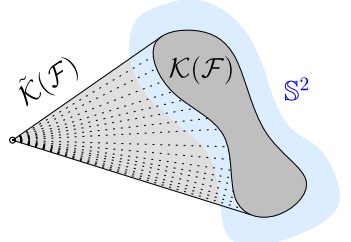

The next lemma gives a simple local geometric interpretation of the tangential cone of a cone of directions of three balls as a cone of directions of at most three attached screens. Since cones of directions live in $\mathbb{S}^{2}$, where defining the tangential cone is awkward, we first pull these objects back to $\mathbb{R}^{3}$ by defining $\tilde{\mathcal{K}}(\mathcal{F})$ as the solid cone in $\mathbb{R}^{3}$ formed by all rays originating from the origin $\mathbf{0}$ and with directions in $\mathcal{K}(\mathcal{F})$. Notice that a direction $\mathbf{u}$ is on the boundary of $\mathcal{K}(\mathcal{F})$ if and only if the ray $\mathbf{0}+\mathbb{R}^{+} \mathbf{u}$ is on the boundary of $\tilde{\mathcal{K}}(\mathcal{F})$, and that $\mathcal{K}(\mathcal{F})$ is smooth at $\mathbf{u}$ if and only if $\tilde{\mathcal{K}}(\mathcal{F})$ is smooth at any point of the ray $\mathbf{0}+\mathbb{R}^{+} \mathbf{u}$, except the origin.

Lemma 10 Let $T$ be a triple of balls in $\mathbb{R}^{3}$, $\mathbf{u}$ a smooth point of $\partial \mathcal{K}(T)$, and $\ell$ the line transversal to $T$ with direction $\mathbf{u}$. Let $S$ be the set of screens defined by $\ell$ and the balls of $T$. Let $\mathbf{p}$ be any point in $\mathbf{0}+\mathbb{R}^{+} \mathbf{u}$ other than $\mathbf{0}$. Then locally near $\mathbf{p}, \tilde{\mathcal{K}}(S)$ coincides with $\mathcal{T}_{\mathbf{p}} \tilde{\mathcal{K}}(T)$, the tangential cone of $\tilde{\mathcal{K}}(T)$ at $\mathbf{p}$. 
Proof Since $\mathbf{u}$ is on the boundary of $\mathcal{K}(T)$, Lemma 3(ii) implies that $T$ has a unique line transversal with direction $\mathbf{u}$, which we denote by $\ell$. We equip $\mathbb{R}^{3}$ with a frame such that $\ell$ is the $z$ axis and let $\mathcal{L}$ denote the set of lines not orthogonal to $\ell$. The map $\psi$ that associates to $\mathbf{x}=\left(x_{1}, x_{2}, x_{3}, x_{4}\right) \in \mathbb{R}^{4}$ the line through the points $\left(x_{1}, x_{2}, 0\right)$ and $\left(x_{3}, x_{4}, 1\right)$ is a homeomorphism from $\mathbb{R}^{4}$ to $\mathcal{L}$, that is, it defines a proper parameterization of $\mathcal{L}$ and $\psi(0, \ldots, 0)=\ell$.

Let $s$ be a screen defined by $\ell$. If $s$ is a plane, then any line in $\mathcal{L}$ intersects it. Otherwise, let $\delta$ denote the boundary of $s$. Let $\mathbf{p}$ and $\mathbf{p}^{\prime}$ be two points on $\delta$. The condition that a line $\tilde{\ell}(\mathbf{x})=\psi\left(x_{1}, x_{2}, x_{3}, x_{4}\right)$ intersects $s$ amounts to evaluating the orientation of a tetrahedron formed by two points from $\tilde{\ell}(\mathbf{x})$ and two points from $\delta$. Since $\delta$ is orthogonal to the $z$ axis, the $z$ coordinates of $\mathbf{p}$ and $\mathbf{p}^{\prime}$ are equal, and this orientation test recasts as a sign condition on the $4 \times 4$ determinant

$$
\left|\begin{array}{cccc}
x_{p^{\prime}}-x_{p} & x_{p} & x_{1} & x_{3} \\
y_{p^{\prime}}-y_{p} & y_{p} & x_{2} & x_{4} \\
0 & z_{p} & 0 & 1 \\
0 & 1 & 1 & 1
\end{array}\right|
$$

which is linear in $x_{1}, \ldots, x_{4}$. Thus, if $L$ is the set of lines in $\mathcal{L}$ that intersect a screen defined by $\ell, \psi^{-1}(L)$ is thus a halfspace in $\mathbb{R}^{4}$ or all of $\mathbb{R}^{4}$. In other words, $\psi^{-1}$ maps the set of line transversals to any screen defined by $\ell$ to $\mathbb{R}^{4}$ or one of its halfspaces.

Now, let $\mathbf{p}$ be a point in $\mathbf{0}+\mathbb{R}^{+} \mathbf{u}$ other than $\mathbf{0}$. Let $S$ denote the set of screens defined by $(\ell, B)$ where $B$ is a ball of $T$ tangent to $\ell$. By Lemma 3(iii), $|S| \leq 3$. Since $\mathbf{u} \in \partial \mathcal{K}(T)$, Lemma 9(ii) implies that $\mathcal{K}(S)$ is, near $\mathbf{u}$, bounded by a great circle. It follows that $\tilde{\mathcal{K}}(S)$ coincides with a halfspace near $\mathbf{p}$. Since $\partial \mathcal{K}(T)$ is smooth at $\mathbf{u}$, the tangential cone to $\tilde{\mathcal{K}}(T)$ at $\mathbf{p}$ is also a halfspace. To prove the statement, it suffices to show that in a neighborhood of $\mathbf{p}$, the interior of $\tilde{\mathcal{K}}(S)$ is contained in $\mathcal{T}_{\mathbf{p}} \tilde{\mathcal{K}}(T)$.

Let $\mathbf{v}$ be a direction in the interior of $\mathcal{K}(S)$. By Lemma 9(ii), the relative interiors of the screens of $S$ have a line transversal $\gamma$. Consider the family of lines $\bar{\ell}(t)=$ $\psi\left(t \psi^{-1}(\gamma)\right)$ that interpolates linearly (in our parameterization of lines) between $\ell$ and $\gamma$. Let $s$ be a screen in $S, b$ the corresponding ball in $T$, and let $\Pi$ denote the plane perpendicular to $\ell$ that contains $s$. We note that the trace of $\bar{\ell}(t)$ on $\Pi$ forms a line passing through $b \cap \ell$. Since $\Pi$ intersects $B$ in a disk tangent to the halfplane $s$ in $b \cap \ell$, it follows that there exists $\epsilon_{s}>0$ such that for any $t \in\left[0, \epsilon_{s}\right]$ the line $\bar{\ell}(t)$ intersects $b$. If $b$ is a ball in $T$ to which $\ell$ is not tangent, the same holds trivially. Thus, there exists $\epsilon>0$ such that if $0 \leq t<\epsilon$, then $\bar{\ell}(t)$ is a line transversal to $T$. The set of directions of the lines $\{\bar{\ell}(t) \mid t \geq 0\}$ forms, in $\mathbb{S}^{2}$, a great circle arc with endpoints $\mathbf{u}$ and $\mathbf{v}$. It follows that $\mathbf{0}+\mathbb{R}^{+} \mathbf{v}$ belongs to the tangential cone of $\tilde{\mathcal{K}}(T)$ at $\mathbf{p}$.

\subsection{Pinning Theorem for Ovaloids}

The proof of Theorem 1 uses one property of cones of triples of balls that may not hold, in general, for cones of triples of ovaloids: that if the boundaries of two such cones of directions intersect in a direction $\mathbf{u}$, there is a neighborhood of $\mathbf{u}$ in which the curves either coincide or intersect only at $\mathbf{u}$. In other words, we use the property that the lower (or upper) envelope of boundaries of cones of directions near one of its 
vertices is defined by at most 2 curves. ${ }^{3}$ To ensure that a similar property holds here, we now deal with semialgebraic ovaloids with bounded description complexity.

We first extend the smoothness condition of Lemma 6 to cones of directions of ovaloids, in view of Lemma 10.

Lemma 11 Let $T$ be a triple of semialgebraic ovaloids in $\mathbb{R}^{3}$. A direction $\mathbf{u}$ is a singular point of $\partial \mathcal{K}(T)$ only if the intersection of the three solid ovaloids is empty and there exists a line with direction $\mathbf{u}$ that is

(i) pinned by $T$, or

(ii) tangent to all three ovaloids, meeting two of them in the same point in which they are externally tangent.

Proof We prove the statement by contraposition. Let $\mathbf{u}$ be a direction of $\partial \mathcal{K}(T), \ell$ the line transversal to $T$ with direction $\mathbf{u}$, and let $\mathbf{p}$ be a point distinct from $\mathbf{0}$ on the ray $\mathbf{0}+\mathbb{R}^{+} \mathbf{u}$. For each ovaloid $C \in T$ we consider a ball $B^{-}(C)$ contained in (resp. $B^{+}(C)$ containing) $C$ such that if $\ell$ is tangent to $C$, then $C$ and $B^{-}(C)$ (resp. $\left.B^{+}(C)\right)$ are internally tangent at $C \cap \ell$, and if $\ell$ intersects the interior of $C$, then $\ell$ also intersects the interior of $B^{-}(C)$ (resp. $B^{+}(C)$ ). We let $T^{-}=\left\{B^{-}(C) \mid C \in T\right\}$ and $T^{+}=\left\{B^{+}(C) \mid C \in T\right\}$. Observe that $\ell$ and $T^{-}$define the same triple of screens as $\ell$ and $T^{+}$; we call $S$ that triple of screens.

We now make two observations. First, note that $\mathbf{u}$ belongs to $\partial \mathcal{K}\left(T^{-}\right)$and $\partial \mathcal{K}\left(T^{+}\right)$, and thus p belongs to $\partial \tilde{\mathcal{K}}\left(T^{-}\right)$and $\partial \tilde{\mathcal{K}}\left(T^{+}\right)$. Second, the inclusions $B^{-}(C) \subseteq C \subseteq B^{+}(C)$ imply that $\mathcal{K}\left(T^{-}\right) \subseteq \mathcal{K}(T) \subseteq \mathcal{K}\left(T^{+}\right)$, and similarly $\tilde{\mathcal{K}}\left(T^{-}\right) \subseteq$ $\tilde{\mathcal{K}}(T) \subseteq \tilde{\mathcal{K}}\left(T^{+}\right)$. As a consequence, $\mathcal{T}_{\mathbf{p}} \tilde{\mathcal{K}}\left(T^{-}\right) \subseteq \mathcal{T}_{\mathbf{p}} \tilde{\mathcal{K}}(T) \subseteq \mathcal{T}_{\mathbf{p}} \tilde{\mathcal{K}}\left(T^{+}\right)$.

Now, by Lemma 10 , we have that near $\mathbf{p}$ the tangential cones $\mathcal{T}_{\mathbf{p}} \tilde{\mathcal{K}}\left(T^{-}\right)$and $\mathcal{T}_{\mathbf{p}} \tilde{\mathcal{K}}\left(T^{+}\right)$both agree with $\tilde{\mathcal{K}}(S)$. By Lemma 9 , we have that near $\mathbf{p}$ the cone $\tilde{\mathcal{K}}(S)$ is a halfspace.

Altogether, we get that near $\mathbf{p}$, the tangential cone $\mathcal{T}_{\mathbf{p}} \tilde{\mathcal{K}}(T)$ is a halfspace. Since $\mathcal{K}(T)$ (and therefore $\tilde{\mathcal{K}}(T)$ ) is semialgebraic, this implies that $\mathbf{p}$ is a smooth point of $\partial \tilde{\mathcal{K}}(T)$. Therefore, $\mathbf{u}$ is a smooth point of $\partial \mathcal{K}(T)$.

We are now ready to prove our extension of Theorem 1 to semialgebraic ovaloids.

Theorem 12 Let $\mathcal{F}$ be a finite family of semialgebraic ovaloids in $\mathbb{R}^{3}$ that pin a line $\ell$. If no two members of $\mathcal{F}$ are externally tangent on $\ell$, then there is a subfamily of $\mathcal{F}$ of size at most 12 that pins $\ell$.

Proof Assume that no triple of $\mathcal{F}$ pins $\ell$, as otherwise the statement is trivially true. Let $\mathbf{u}$ denote the direction of $\ell$. As noted in Sect. 2.2, Lemma 2 and Lemma 3(ii) immediately extend to ovaloids. It follows that a family $\mathcal{F}$ of ovaloids pins a line $\ell$ if and only if the direction of $\ell$ is an isolated point of $\mathcal{K}(\mathcal{F})=\bigcap_{T \in\left(\begin{array}{c}\mathcal{F} \\ 3\end{array}\right)} \mathcal{K}(T)$. Now, for every triple $T \subset \mathcal{F}$ such that $\mathbf{u}$ is on the boundary of $\mathcal{K}(\mathcal{F})$, Lemma 11 ensures that $\partial \mathcal{K}(T)$ is smooth at $\mathbf{u}$. We can then, as in the proof of Theorem 1 , recast $\mathcal{K}(\mathcal{F})$ near

\footnotetext{
${ }^{3}$ The constant 2 is not crucial: any constant bound $k$ would imply a pinning theorem with constant $6 k$.
} 
$\mathbf{u}$ as the region above the lower envelope and below the upper envelope of a family of semialgebraic functions. Locally, these upper and lower envelopes are defined by two curves each, and the statement follows.

\section{References}

1. Agarwal, P.K., Aronov, B., Sharir, M.: Line transversals of balls and smallest enclosing cylinders in three dimensions. Discrete Comput. Geom. 21(3), 373-388 (1999)

2. Ambrus, G., Bezdek, A., Fodor, F.: A Helly-type transversal theorem for $n$-dimensional unit balls. Arch. Math. 86(5), 470-480 (2006)

3. Aronov, B., Cheong, O., Goaoc, X., Rote, G.: Lines pinning lines. Discrete Comput. Geom. (2010). 10.1007/s00454-010-9288-6

4. Basu, S., Pollack, R., Roy, M.-F.: Algorithms in Real Algebraic Geometry, 2nd edn. Algorithms and Computation in Mathematics, vol. 10. Springer, Berlin (2003)

5. Borcea, C., Goaoc, X., Petitjean, S.: Line transversals to disjoint balls. Discrete Comput. Geom. 1-3, 158-173 (2008)

6. Cheong, O., Goaoc, X., Holmsen, A.: Lower bounds to Helly numbers of line transversals to disjoint congruent balls. Isr. J. Math. (to appear)

7. Cheong, O., Goaoc, X., Holmsen, A., Petitjean, S.: Hadwiger and Helly-type theorems for disjoint unit spheres. Discrete Comput. Geom. 1-3, 194-212 (2008)

8. Danzer, L.: Über ein Problem aus der kombinatorischen Geometrie. Arch. Math. 8(5), 347-351 (1957)

9. Danzer, L., Grünbaum, B., Klee, V.: Helly's theorem and its relatives. In: Klee, V. (ed.) Convexity, Proc. of Symposia in Pure Math, pp. 101-180. Am. Math. Soc., Providence (1963)

10. Eckhoff, J.: Helly, Radon and Caratheodory type theorems. In: Goodman, J. E., O'Rourke, J. (eds.) Handbook of Convex Geometry, pp. 389-448. North-Holland, Amsterdam (1993)

11. Goaoc, X.: Some discrete properties of the space of line transversals to disjoint balls. In: Emiris, I., Sottile, F., Theobald, T. (eds.) Non-Linear Computational Geometry. The IMA Volumes in Mathematics and Its Applications, vol. 151, pp. 51-83. Springer, Berlin (2010)

12. Goodman, J.E., Pollack, R., Wenger, R.: Geometric transversal theory. In: Pach, J. (ed.) New Trends in Discrete and Computational Geometry. Algorithms and Combinatorics, vol. 10, pp. 163-198. Springer, Heidelberg (1993)

13. Holmsen, A.: Recent progress on line transversals to families of translated ovals. In: Goodman, J.E., Pach, J., Pollack, R. (eds.) Computational Geometry-Twenty Years Later. Am. Math. Soc., Providence (2008)

14. Holmsen, A., Katchalski, M., Lewis, T.: A Helly-type theorem for line transversals to disjoint unit balls. Discrete Comput. Geom. 29, 595-602 (2003)

15. Holmsen, A., Matoušek, J.: No Helly theorem for stabbing translates by lines in $\mathbb{R}^{d}$. Discrete Comput. Geom. 31, 405-410 (2004)

16. Montiel, S., Ros, A., Babbitt, D.G.: Curves and Surfaces, 2nd edn. Graduate Studies in Mathematics, vol. 69. AMS, Providence (2009)

17. Rauch, J.: An inclusion theorem for ovaloids with comparable second fundamental forms. J. Differ. Geom. 9(4), 501-505 (1974)

18. Vincensini, P.: Figures convexes et variétés linéaires de l'espace euclidien à $n$ dimensions. Bull. Sci. Math. 59, 163-174 (1935)

19. Wenger, R.: Helly-type theorems and geometric transversals. In: Goodman, J. E., O’Rourke, J. (eds.) Handbook of Discrete \& Computational Geometry, 2nd edn., pp. 73-96. CRC Press, Boca Raton (2004), Chap. 4 